\title{
Heat transfer between a fluid-saturated porous medium and a permeable wall with fluid injection or withdrawal
}

\author{
H. J. H. BROUWERS \\ University of Twente, Department of Civil Engineering \& Management, P.O. Box 217, 7500 AE Enschede, \\ The Netherlands
}

(Received 16 June 1993 and in final form 27 September 1993)

\begin{abstract}
The present paper addresses heat and mass transfer between a permeable wall and a fluidsaturated porous medium. To assess the effect of wall suction or injection on sensible heat transfer, a stagnant film model is developed. The model yields a thermal correction factor accounting for the effect of wall transpiration on heat transfer. In order to apply this correction factor, neutral (or zero mass transfer) heat transfer rates must be specified. In the past, for a large number of practical situations heat transfer correlations have been obtained with the boundary layer model, which are summarized here. Subsequently, the derived correction factor is applied to free, mixed and forced convection flows along vertical and horizontal permeable walls embedded in a porous medium. The predictions of the film model are compared and found to be in good agreement with corresponding results obtained with the boundary layer model (also provided by the literature). Hence, the film model approach appears to be a compact and adequate description of the processes considered.
\end{abstract}

\section{INTRODUCTION}

FORCED, mixed, and free convection flows and heat transfer in fluid-saturated porous media are encountered in many geophysical and engineering applications. Geophysical applications are thermally enhanced oil-recovery, energy storage, pore water convection near salt domes (for the storage of nuclear wastes) and movement of contaminants in ground water. Some of the direct technological examples of heat transfer in porous media are drying processes, powder metallurgy, transpiration cooling, insulation of buildings and pipes, and chemical catalytic reactors.

This wide spectrum of interests has led to a large volume of investigations in this field in the last 50 years. Most investigations issue from Darcian fluid flow, named after Darcy [1], which is extended with nonisothermal buoyancy cffects by invoking the Oberbeck-Boussinesq approximations. $\dagger$ The possibility of free convection in a porous material was first pointed out by Horton and Roger [5] and Lapwood [6]. Wooding [7, 8] conducted the first numerical and experimental studies on convection in porous layers. Later these studies have been continued by among others McNabb [9], Prats [10], Elder [11], Combarnous and Bia [12], Cheng and Chang [13], Cheng and Minkowycz [14], Cheng [15, 16], Renken and Poulikakos [17, 18] and Lai and Kulacki [19].

+ Though thesc approximations are generally attributed to Boussinesq [2], they were originally set out by Oberbeck [3]. A historical review is found in Joseph [4].
All mentioned studies concerned flow in a porous medium bounded by a flat impermeable wall. In some practical cases however, fluid injection or withdrawal (or suction) though the wall is encountered. From a geothermal power plant, for example, the residual hot water is usually disposed of via subsurface injection wells which can be modeled as a permeable wall. The effect of wall transpiration on flow in porous media and convective heat transfer has been examined numerically by Cheng [20], Minkowycz et al. [21] and Lai and Kulacki [22].

A powerful approach for describing the effect of wall transpiration on heat transfer is offered by the classical film model. This model provides general analytical expressions, the so-called correction factors, which can be applied to any system of importance. In the past these film model correction factors have been successfully used to predict the effect of surface mass transfer on free fluid transport phenomena. Recent reviews of the film theory are found in Bannwart [23], Bannwart and Bontemps [24], Brouwers [25] and Brouwers and Chesters [26].

To the author's knowledge, the film model has never been derived and employed on convective heat transfer between a permeable wall and a fluidsaturated porous medium. Hence, from an analysis of a porous film a correction factor for the effect of wall transpiration on heat transfer is derived. Furthermore, this correction is applied to forced, mixed and free convective flow along vertical and horizontal plates embedded in a porous medium (mixed convection constitutes the interface between pure free (or natural) and pure forced convection). Next, the impermeable wall Nusselt number correlations are 


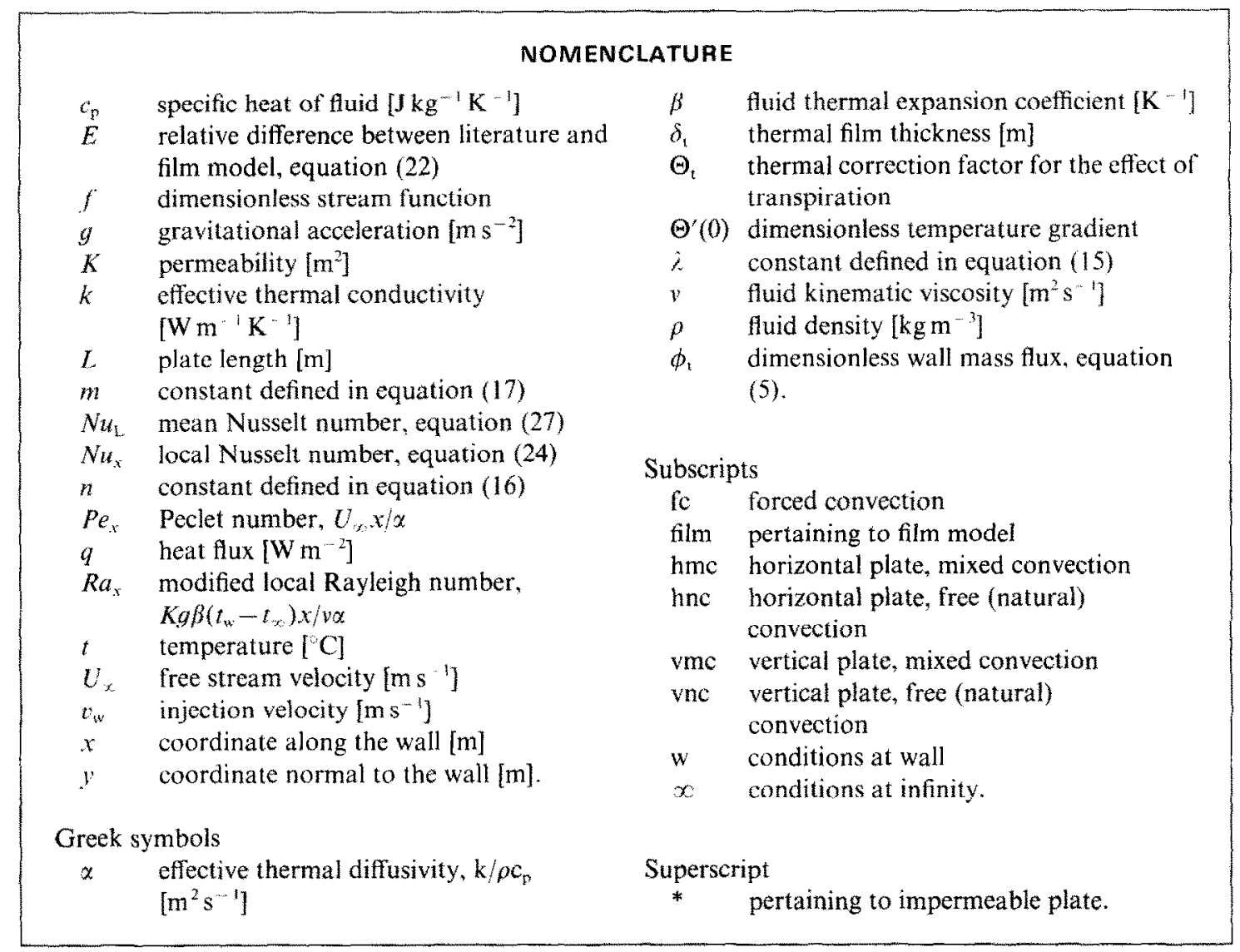

summarized, provided by the literature. These correlations are needed in order to apply the film model correction factor. Subsequently, the predictions of the compact film model expression are compared with the permeable wall results of Cheng [20] and Lai and Kulacki [22], which proceed from a numerical similarity solution of the continuity, the momentum (Darcy) and the energy equation.

\section{POROUS FILM MODEL}

Consider a film consisting of a fluid-saturated porous medium next to a permeable wall (Fig. 1). Through this film there is a transport of fluid which is injected or withdrawn through the wall. Neglecting viscous dissipation, heat sources, radiation and considering a steady situation the energy equation of this film reads :

$$
\rho c_{\mathrm{p}} v_{w} \frac{\mathrm{d} t}{\mathrm{~d} y}=k \frac{\mathrm{d}^{2} t}{\mathrm{~d} y^{2}}
$$

At the wall the temperature reads :

$$
t(y=0)=t_{\mathrm{w}} .
$$

At the opposite side of the porous layer the bulk temperature attained is :

$$
t\left(y=\delta_{1}\right)=t_{t: 2}
$$

Solving equation (1) and applying boundary conditions (2) and (3) yields the temperature profile in the film:

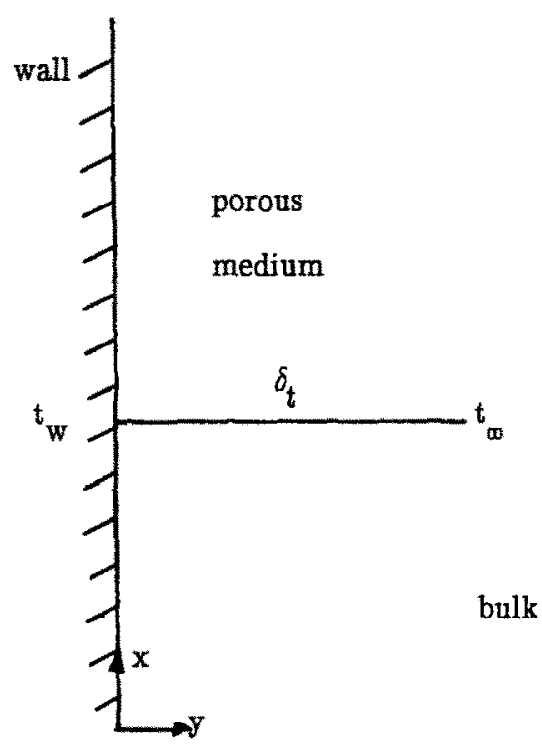

Frg. 1. Porous film 


$$
t(y)=\left(t_{x}-t_{\mathrm{w}}\right)\left(\frac{\mathrm{e}^{-\left(y \phi_{\mathrm{t}} / \delta_{1}\right)}-1}{\mathrm{e}^{-\phi_{\mathrm{t}}}-1}\right)+t_{\mathrm{w}}
$$

where $\phi_{\mathrm{t}}$ represents the dimensionless thermal mass flux :

$$
\phi_{\mathrm{t}}=-\frac{\rho v_{\mathrm{w}} c_{\mathrm{p}} \delta_{1}}{k}
$$

The heat transfer from the fluid-saturated medium to the wall is described by Fourier's law as:

$$
q=\left.k \frac{\mathrm{d} t}{\mathrm{~d} y}\right|_{y=0}=\frac{k}{\delta_{\mathrm{t}}} \Theta_{1, \mathrm{tim}}\left(t_{\infty}-t_{\mathrm{w}}\right),
$$

wherein equation (4) has been inserted. In cquation (6) the following factor has been introduced:

$$
\Theta_{\mathrm{t}, \mathrm{fim}}=\left(\frac{-\phi_{\mathrm{t}}}{\mathrm{e}^{-\phi_{\mathrm{i}}}-1}\right) \text {. }
$$

For heat transfer from medium to wall without wall transpiration (i.e. $v_{\mathrm{w}} \rightarrow 0$ or $\phi_{\mathrm{t}} \rightarrow 0$ ) $\Theta_{\mathrm{t}, \text { film }}$ tends to unity. Note that this factor is analogous to the expression derived from the customary pure fluid film model, where this factor is referred to as the Ackermann correction [25].

In the case of convective heat transfer, according to the film theory approach, the actual local Nusselt number, denoted by $N u_{x}$, in the presence of mass transfer follows from multiplying the zero mass transfer (or neutral) Nusselt number, denoted as $N u_{x}^{*}$, by $\Theta_{\mathrm{t}, \mathrm{nim}}$ :

$$
N u_{x}=\Theta_{\text {t.film }} N u_{x}^{*}
$$

The thermal film thickness is then taken to be as :

$$
\delta_{\mathrm{t}}=\frac{x}{N u_{x}^{*}}
$$

where $x$ is a coordinate along the wall (in the direction of flow). The local-impermeable plate-Nusselt number depends on the convective system considered. Some frequently occurring cases are discussed in the following section.

One can readily conclude from equations $(5),(7)$ (9) that, following the film model, the effect of a surface mass flux on heat transfer can be assessed in a relatively simple way. Furthermore, though the presented analysis is based on an imposed wall mass flux, the same correction factor will follow when the mass flux originates from diffusion. Hence the correction factor derived here can be applied to porous media with heat transfer in the presence of a diffusional mass flux (by wall condensation or evaporation) as well.

\section{IMPERMEABLE PLATE NUSSELT NUMBERS}

In this section Nusselt number correlations are summarized for forced, mixed and free convective heat transfer to both vertical and horizontal impermeable plates. These correlations are the results of investigations of previous researchers. All analyses were based on a numerical analysis of Darcian flow in a saturated porous material with invoked boundary layer conditions. The fluid was considered to conduct as Newtonian and incompressible.

For forced convection over an impermeable plate embedded in a porous medium the local neutral Nusselt number reads:

$$
N u_{x}^{*}=-\Theta^{\prime}(0)_{\mathrm{fc}}^{*} P e_{x^{\prime}}^{1 / 2}
$$

see Cheng [15]. The constant factor on the right-hand side of this equation depends on the wall temperature and temperature distribution. For constant fluid velocity along an isothermal plate it amounts to 0.5641 , in the case of constant heat flux it has a value of 0.8540 .

For combined forced and free convective flow along a horizontal or vertical impermeable surface in a porous material the local Nusselt number depends on both the Peclet and Rayleigh number. For a horizontal plate it reads :

$$
N u_{x}^{*}=-\Theta^{\prime}(0)_{\mathrm{hmc}}^{*} P e_{x}^{1 / 2},
$$

see Cheng [16] and for a vertical plate the Nusselt number follows from:

$$
N u_{x}^{*}=-\Theta^{\prime}(0)_{v m c}^{*} P e_{x^{1 / 2}}
$$

see Cheng [15]. The constant in equation (11) depends on the bulk velocity, the temperature distribution of the plate, and $R a_{x} / P e_{x}^{3 / 2}$. For mixed convection along a vertical plate the constant in equation (12) depends on the temperature distribution, $R a_{x} / P e_{x}$, and the directions of buoyancy and free stream velocity (aiding or opposing).

The local Nusselt number for pure free convective heat transfer to a horizontal nonisothermal plate in a porous medium follows from Cheng and Chang [13] as:

$$
N u_{x}^{*}=-\Theta^{\prime}(0)_{h \mathrm{hc}}^{*} R a_{x}^{1 / 3} .
$$

For an impermeable vertical plate in a porous material the local Nusselt number has been obtained by Cheng and Minkowycz [14] as:

$$
N u_{x}^{*}=-\Theta^{\prime}(0)_{\mathrm{vic}}^{*} R a_{x}^{1 / 2} .
$$

Both $\Theta^{\prime}(0)_{\text {hnc }}^{*}$ and $\Theta^{\prime}(0)_{\text {vnc }}^{*}$ depend on the thermal boundary conditions at the plate surface. For an isothermal plate $\Theta^{\prime}(0)_{\mathrm{vnc}}^{*}$ has a value of -0.4440 , while for a constant heat flux $\Theta^{\prime}(0)_{\mathrm{vnc}}^{*}$ and $\Theta^{\prime}(0)_{\mathrm{nnc}}^{*}$ amount to -0.6788 and -0.8164 , respectively.

Experimental results of Elder [11], Combarnous and Bia [12], Renken and Poulikakos [18] and Lai and Kulacki [19] are in agreement with theoretical predictions. These predictions were based on a boundary layer analysis of mixed convective heat transfer in horizontal porous layers next to impermeable walls. Recently Renken and Poulikakos [17] reported the agreement between (Darcian) theory, based again on the boundary layer model, and forced convective heat transfer experiments for small porous medium Reynolds numbers. A possible agreement between the boundary layer model and film model will there- 
Table 1. Numerical results of Lai and Kulacki [22] and the film model for the effect of transpiration on forced convective heat transfer ( $q_{w}$ is constant)

\begin{tabular}{|c|c|c|c|c|c|}
\hline \multicolumn{2}{|c|}{ Lai and Kulacki [22 } & & & & \\
\hline & $\ldots \ldots$ & equation (20) & equation (2l) & equation (7) & cquation (22) \\
\hline$f_{w}$ & $-\Theta^{\prime}(0)$ & $\Theta_{t, k}$ & $\phi_{1, k}$ & $\Theta_{1 . \text { filtut }}$ & $E_{\mathrm{i}}$ \\
\hline-1.0 & 0.6337 & 0.7151 & -0.5642 & 0.7443 & $4.1 \%$ \\
\hline 0.0 & 0.8862 & 1.0000 & 0.0000 & 1.0000 & $0.0 \%$ \\
\hline 1.0 & 1.2009 & 1.3551 & 0.5642 & 1.3085 & $-3.4 \%$ \\
\hline
\end{tabular}

fore, for the above mentioned cases, also imply an agreement between film model and practice.

\section{FORCED AND MIXED CONVECTION ALONG PERMEABLE PLATES}

The film model correction for the effect of mass transfer on heat transfer is compared with the results of previous investigators. All these foregoing studies concern forced and mixed convective boundary layer flow over flat vertical plates embedded in porous materials with imposed transpiration.

The effect of mass transfer through a permeable horizontal plate in a porous medium on pure forced and mixed convective heat transfer has been investigated by Lai and Kulacki [22]. These investigators obtained similarity solutions for the special case where the surface temperature:

$$
t_{\mathrm{w}}=t_{s}+C_{1} x^{\lambda}
$$

the wall transpiration velocity:

$$
v_{w}=C_{2} x^{\prime \prime},
$$

and the free stream velocity:

$$
U_{x}=C_{3} x^{\prime \prime \prime} \text {, }
$$

are all prescribed power functions of the coordinate along the plate. It was shown by Lai and Kulacki [22] that similarity solutions exist for:

$$
\begin{aligned}
& \lambda=\frac{3 m+1}{2}, \\
& n=\frac{m-1}{2} .
\end{aligned}
$$

For a number of dimensionless surface mass flux parameters $f_{\mathrm{w}}$ the governing equations were solved and hence the dimensionless temperature gradient at the wall, denoted by $\Theta^{\prime}(0)$, was obtained. The effect of mass transfer on heat transfer can simply be expressed by:

$$
\Theta_{1, f \in}=\frac{N u}{N u^{*}}=\frac{\Theta^{\prime}(0)}{\Theta^{\prime}(0)^{*}},
$$

which can be compared to the correction factor of the film model. The parameter $\phi_{\mathrm{L}}$ is therefore expressed in terms of $f_{\mathrm{w}}$ by equations (5), (9), (10), (16), (17), (19) and equation (14) of Lai and Kulacki [22] :

$$
\phi_{\mathrm{L} . \mathrm{c} \mathrm{c}}=\begin{aligned}
& f_{\mathrm{w}}(\lambda+1) \\
& 3 \Theta^{\prime}(0)_{\mathrm{c}}^{*}
\end{aligned} .
$$

In Table 1 for an injection case $\left(f_{\mathrm{w}}=-1\right)$, a neutral case $\left(f_{\mathrm{w}}=0\right)$, and a suction case $\left(f_{\mathrm{w}}=1\right)$ the values of $\Theta^{\prime}(0)$ and $\Theta_{1, f i}$ are listed for pure forced convective flow, whereby $\lambda=1 / 2, m=0$ and $n=-1 / 2$. Following ref. [22] these values correspond to a constant heat flux problem. In Table 1 also $\phi_{\text {t.fc }}$ and $\Theta_{\text {i.film }}$ are included, determined with equations (21) and (7), respectively. The relative difference between film model prediction and similarity solution is assessed by:

$$
E_{\mathrm{lc}}=\frac{\Theta_{\mathrm{t} . \mathrm{fllm}}-\Theta_{\mathrm{t} . \mathrm{f}_{\mathrm{c}}}}{\widetilde{\Theta_{\mathrm{t} . \mathrm{jc}}}}
$$

which is also included in Table 1. Table 2 has same properties listed, but now for $\lambda-2, m-1$ and $n=0$. These values correspond to the case of constant transpiration velocity, see equation (16).

Tables 1 and 2 indicate the excellent predictions of the effect of mass transfer on heat transfer by the film model. The relative difference between film model and numerical results in ref. [22], governed by $E_{\mathrm{fc}}$, is less than $5 \%$ for $\left|\phi_{1, \mathrm{~d}}\right| \leqslant 0.5642$. A similar accuracy has been obtained by Mickley et al. [27] for forced convective boundary layer flow over a flat permeable plate.

As said above, Lai and Kulacki [22] examined combined forced and free convection over a horizontal plate with wall transpiration as well. In Table 3 their mixed convective $\Theta^{\prime}(0)$ and resulting $\Theta_{\text {t.hmc }}$ are tabulated for $i=1 / 2, m=0$ and $n=-1 / 2$ and $R a_{x} /$ $P e_{x}^{3 / 2}=1$, implying a constant wall heat flux. $R a_{x}$ / $P e_{x}^{3 / 2}=1$ concerns a situation where both the pressure gradient and the buoyancy gradient contribute to flow and heat transfer to a similar extent. Hence, this ratio corresponds to a true mixed convection problem. In Table 4 the pertaining results are listed for $\lambda=2$, $m=1$ and $n=0$, which is to say a constant surface mass flux. The computed values of $E_{\mathrm{hme}}$ which are listed in Tables 3 and 4 illustrate again the accurate predictions of the basic film model with respect to the effect of surface transpiration on mixed convective heat transfer between a fluid in a porous material and a flat horizontal plate.

A similar analysis has, to the author's knowledge, not been carried out yet for mixed convection over vertical plates. Consequently, no numerical results of this case are available to compare the film model with.

\section{FREE CONVECTION ALONG PERMEABLE PLATES}

Free (or natural) convective flow along permeable plates embedded in a porous material has been inves- 
Table 2. Numerical results of Lai and.Kulacki [22] and the film model for the effect of transpiration on forced convective heat transfer ( $v_{w}$ is constant)

\begin{tabular}{|c|c|c|c|c|c|}
\hline \multicolumn{2}{|c|}{ Lai and Kulacki [22] } & \multirow{2}{*}{$\begin{array}{c}\text { equation }(20) \\
\Theta_{\mathrm{t} . \mathrm{fc}}\end{array}$} & \multirow{2}{*}{$\begin{array}{c}\text { equation (21) } \\
\phi_{\mathrm{t} . \mathrm{fc}}\end{array}$} & \multirow{2}{*}{$\begin{array}{c}\text { equation (7) } \\
\Theta_{\mathrm{t}, \mathrm{film}}\end{array}$} & \multirow{2}{*}{$\begin{array}{c}\text { equation (22) } \\
E_{\mathrm{fc}}\end{array}$} \\
\hline$f_{\mathrm{w}}$ & $-\Theta^{\prime}(0)$ & & & & \\
\hline-1.0 & 1.1258 & 0.7055 & -0.6267 & 0.7192 & $1.9 \%$ \\
\hline 0.0 & 1.5957 & 1.0000 & 0.0000 & 1.0000 & $0.0 \%$ \\
\hline 1.0 & 2.2117 & 1.3860 & 0.6267 & 1.3459 & $-2.9 \%$ \\
\hline
\end{tabular}

Table 3. Numerical results of Lai and Kulacki [22] and the film model for the effect of transpiration on mixed convective heat transfer to a horizontal plate $\left(R a_{x} / P e_{x}^{3,2}-1, q_{\mathrm{w}}\right.$ is constant $)$

\begin{tabular}{cccccc}
\hline \multicolumn{2}{c}{ Lai and Kulacki [22] } & & & \\
equation (20) \\
$f_{\mathrm{w}}$ & $-\Theta^{\prime}(0)$ & $\Theta_{\mathrm{thmc}}$ & $\begin{array}{c}\text { equation (21) } \\
\phi_{\mathrm{thmc}}\end{array}$ & $\begin{array}{c}\text { equation (7) } \\
\Theta_{\mathrm{t}, \mathrm{hlilm}}\end{array}$ & $\begin{array}{c}\text { equation (22) } \\
E_{\mathrm{hmc}}\end{array}$ \\
\hline-1.0 & 0.8862 & 0.8042 & -0.4537 & 0.7902 & $-1.7 \%$ \\
0.0 & 1.1020 & 1.0000 & 0.0000 & 1.0000 & $0.0 \%$ \\
1.0 & 1.3745 & 1.2473 & 0.4537 & 1.2439 & $-0.3 \%$ \\
\hline
\end{tabular}

tigated by Cheng [20], Minkowycz et al. [21] and Lai and Kulacki [22].

Cheng [20] studied the effect of the lateral mass flux on free convective boundary layer flow along a vertical plate in a porous layer. Similarity solutions were obtained, the temperature (equation (15)) and transpiration velocity (equation (16)) described as power functions of the vertical coordinate, for :

$$
n=\frac{\lambda-1}{2}
$$

For various dimensionless surface transpiration parameters $f_{\mathrm{w}}$ the dimensionless wall temperature gradient, denoted by $\Theta^{\prime}(0)$, was obtained. The effect of mass transfer on heat transfer is characterized again by the ratio of transpiration heat flux and neutral (impermeable plate) heat flux, see equation (20).

The dimensionless variables $f_{\mathrm{w}}$ and $\phi_{1, \mathrm{vuc}}$ are related by equations (5), (9), (14)-(16), (23),

$$
N u_{x}=\frac{\left.x \frac{\mathrm{d} t}{\mathrm{~d} y}\right|_{y=0}}{t_{x}-t_{\mathrm{w}}},
$$

and equation (16) of ref. [20], resulting in

$$
\phi_{\mathrm{t}, \mathrm{vnc}}=\frac{f_{\mathrm{w}}(\lambda+1)}{2 \Theta^{\prime}(0)_{\mathrm{vnc}}^{*}} .
$$

In Tables 5-7 various $f_{\mathrm{w}}, \Theta^{\prime}(0)$ and $\Theta_{\mathrm{t}, \mathrm{vnc}}$ are listed, as well as the corresponding $\phi_{1, \text { vnc }}$ and $\Theta_{1, \text { illm }}$, for $\lambda=0$ (isothermal plate), $\lambda=1 / 3$ (uniform heat flux) and $\lambda=1$ (uniform transpiration). The tables disclose that the general agreement between film model and similarity solution is good, and highest for suction (i.e. $\left.f_{\mathrm{w}}>0, \phi_{\mathrm{t}, \mathrm{vnc}}>0\right)$. Note that a comparable agreement between film model and numerical results was found by Brouwers [28] for free convection along a permeable vertical plate placed in a viscous fluid.

Minkowycz et al. [21] and Lai and Kulacki [22] investigated the effect of surface mass flux on free convection flow adjacent to horizontal plates. The former investigators obtained numerical solutions with a local non-similarity method in the case of constant surface mass flux and various power-law variations of the wall temperature, see equation (10).

Lai and Kulacki [22] showed that similarity solutions are permitted for:

$$
n=\frac{\hat{\imath}-2}{3},
$$

which is equivalent to equations (18) and (19). For several dimensionless surface mass flux parameters $f_{\mathrm{w}}$ the dimensionless temperature gradient at the wall $\Theta^{\prime}(0)$ as obtained with the similarity solution method. The correction factor for the effect of mass transfer on heat transfer is defined by equation (20), which is comparable with the film model correction factor.

Table 4. Numerical results of Lai and Kulacki [22] and the film model for the effect of transpiration on mixed convective heat transfer to a horizontal plate $\left(R a_{x} / P e_{x}^{3 / 2}=1, v_{w}\right.$ is constant $)$

\begin{tabular}{rccccc}
\hline \multicolumn{2}{c}{ Lai and Kulacki [22] } & & & & \\
requation (20) & equation (21) \\
$f_{\mathrm{w}}$ & $-\Theta^{\prime}(0)$ & $\Theta_{\mathrm{t}, \mathrm{hmc}}$ & $\begin{array}{c}\text { equation (7) } \\
\Theta_{\mathrm{t}, \mathrm{him}}\end{array}$ & $\begin{array}{c}\text { equation (22) } \\
E_{\mathrm{hmc}}\end{array}$ \\
\hline-1.0 & 1.6309 & 0.8137 & -0.4989 & 0.7712 & $-5.2 \%$ \\
0.0 & 2.0044 & 1.0000 & 0.0000 & 1.0000 & $0.0 \%$ \\
1.0 & 2.5182 & 1.2563 & 0.4989 & 1.2701 & $1.1 \%$ \\
\hline
\end{tabular}


Table 5. Numerical results of Cheng [20] and the film model for the eflect of transpiration on free convective heat transfer to a vertical plate ( $t_{\omega}$ is constant)

\begin{tabular}{|c|c|c|c|c|c|}
\hline \multicolumn{2}{|c|}{ Cheng [20] } & \multirow{2}{*}{$\begin{array}{c}\text { equation }(20) \\
\Theta_{1}\end{array}$} & \multirow{2}{*}{$\begin{array}{c}\text { equation (25) } \\
\phi_{t, r n c}\end{array}$} & \multirow{2}{*}{$\begin{array}{c}\text { equation (7) } \\
\Theta_{\text {t.film }}\end{array}$} & \multirow{2}{*}{$\begin{array}{c}\text { equation (22) } \\
E_{\text {vns }}\end{array}$} \\
\hline$f_{\mathrm{w}}$ & $-\Theta^{\prime}(0)$ & & & & \\
\hline-10 & 02043 & 0.4603 & -1266 & 0.5403 & $174 \%$ \\
\hline-0.8 & 0.2432 & 0.5480 & -0.9013 & 0.6161 & $12.4 \%$ \\
\hline-0.6 & 0.2865 & 0.6456 & -0.6760 & 0.6998 & $8.4 \%$ \\
\hline-0.4 & 0.3345 & 0.7537 & -0.4507 & 0.7915 & $5.0 \%$ \\
\hline-0.2 & 0.3870 & 0.8720 & -0.2253 & 0.8916 & $2.2 \%$ \\
\hline 0.0 & 0.4438 & 1.0000 & 0.0000 & 1.0000 & $0.0 \%$ \\
\hline 0.2 & 0.5050 & 1.1379 & 0.2253 & 1.1169 & $-1.8 \%$ \\
\hline 0.4 & 0.5701 & 1.2846 & 0.4507 & 1.2422 & $-3.3 \%$ \\
\hline 0.6 & 0.6389 & 1.4396 & 0.6760 & 1.3758 & $-4.4 \%$ \\
\hline 0.8 & 0.7111 & 1.6023 & 0.9013 & 1.5174 & $-5.3 \%$ \\
\hline 1.0 & 0.7863 & 1.7717 & 1.1266 & 1.6669 & $-5.9 \%$ \\
\hline
\end{tabular}

Table 6. Numerical results of Cheng [20] and the film model for the effect of transpiration on free convective heat transfer to a vertical plate ( $q_{w}$ is constant)

\begin{tabular}{|c|c|c|c|c|c|}
\hline \multicolumn{2}{|c|}{ Cheng [20] } & \multirow{2}{*}{$\begin{array}{c}\text { equation }(20) \\
\Theta_{i, v n c}\end{array}$} & \multirow{2}{*}{$\begin{array}{c}\text { equation (25) } \\
\phi_{1 . \mathrm{vnc}}\end{array}$} & \multirow{2}{*}{$\begin{array}{c}\text { equation (7) } \\
\Theta_{\mathrm{t}, \mathrm{filt}}\end{array}$} & \multirow{2}{*}{$\begin{array}{c}\text { equation (22) } \\
E_{\mathrm{vnc}}\end{array}$} \\
\hline$f_{w}$ & $-\Theta^{\prime}(0)$ & & & & \\
\hline-1.0 & 0.3971 & 0.5860 & -0.9839 & 0.5874 & $0.2 \%$ \\
\hline-0.8 & 0.4416 & 0.6517 & -0.7871 & 0.6576 & $0.9 \%$ \\
\hline-0.6 & 0.4917 & 0.7256 & -0.5903 & 0.7337 & $1.1 \%$ \\
\hline-0.4 & 0.5476 & 0.8081 & -0.3935 & 0.8161 & $1.0 \%$ \\
\hline-0.2 & 0.6096 & 0.8996 & -0.1968 & 0.9048 & $0.6 \%$ \\
\hline 0.0 & 0.6776 & 1.0000 & 0.0000 & 1.0000 & $0.0 \%$ \\
\hline 0.2 & 0.7517 & 1.1094 & 0.1968 & 1.1016 & $-0.7 \%$ \\
\hline 0.4 & 0.8316 & 1.2273 & 0.3935 & 1.2096 & $-1.4 \%$ \\
\hline 0.6 & 0.9169 & 1.3532 & 0.5903 & 1.3240 & $-2.1 \%$ \\
\hline 0.8 & 1.007 & 1.486 & 0.7871 & 1.4447 & $-2.8 \%$ \\
\hline 1.0 & 1.102 & 1.626 & 0.9839 & 1.5713 & $-3.4 \%$ \\
\hline
\end{tabular}

The dimensionless properties $f_{\mathrm{w}}$ and $\phi_{\mathrm{t} . \mathrm{hnc}}$ are related by equation (21). This relation follows readily from combining cquations (5), (9), (13), (15), (16), (26) and equation (13) of ref. [22].

In Tables 8 and 9 various $f_{\mathrm{w}}, \Theta^{\prime}(0)$ and $\Theta_{\text {t.hne }}$ are listed, as well as the corresponding $\phi_{\text {l.hne }}$ and $\Theta_{\text {t.film }}$, for $\lambda=1 / 2$ (constant heat flux) and $\lambda=2$ (uniform transpiration), respectively. The tabulated small $E_{\mathrm{hnc}}$ disclose again that the film model and similarity solution agree very well. A similar agreement between film model and numerical results was found by Brouwers [29] for free convective heat transfer to/from a horizontal permeable plate in a viscous fluid.

\section{MEAN HEAT TRANSFER}

In the foregoing the attention has been focused on the effect of mass transfer on local heat transfer between the porous medium and wall. In what follows the effect on mean heat transfer is analyzed in some detail.

Table 7. Numerical results of Cheng [20] and the film model for the effect of transpiration on free convective heat transfer to a vertical plate ( $v_{w}$ is constant)

\begin{tabular}{|c|c|c|c|c|c|}
\hline \multicolumn{2}{|c|}{ Cheng [20] } & \multirow{2}{*}{$\begin{array}{c}\text { equation }(20) \\
\Theta_{\text {t.unc }}\end{array}$} & \multirow{2}{*}{$\begin{array}{c}\text { equation (25) } \\
\phi_{\text {t.vnc }}\end{array}$} & \multirow{2}{*}{$\begin{array}{c}\text { equation }(7) \\
\Theta_{1, \text { film }}\end{array}$} & \multirow{2}{*}{$\begin{array}{c}\text { equation (22) } \\
E_{\text {เnc }}\end{array}$} \\
\hline$f_{\mathrm{w}}$ & $-\Theta^{\prime}(0)$ & & & & \\
\hline-1.0 & 0.6180 & 0.6180 & -1.0000 & 0.5820 & $-5.8 \%$ \\
\hline-0.8 & 0.6770 & 0.6770 & -0.8000 & 0.6528 & $-3.6 \%$ \\
\hline-0.6 & 0.7440 & 0.7440 & -0.6000 & 0.7298 & $-1.9 \%$ \\
\hline-0.4 & 0.8198 & 0.8198 & -0.4000 & 0.8133 & $-0.8 \%$ \\
\hline-0.2 & 0.9049 & 0.9049 & -0.2000 & 0.9033 & $-0.2 \%$ \\
\hline 0.0 & 1.000 & 1.0000 & 0.0000 & 1.0000 & $0.0 \%$ \\
\hline 0.2 & 1.104 & 1.104 & 0.2000 & 1.1033 & $-0.1 \%$ \\
\hline 0.4 & 1.219 & 1.219 & 0.4000 & 1.2133 & $-0.5 \%$ \\
\hline 0.6 & 1.344 & 1.344 & 0.6000 & 1.3298 & $-1.1 \%$ \\
\hline 0.8 & 1.477 & 1.477 & 0.8000 & 1.4528 & $-1.6 \%$ \\
\hline 1.0 & 1.618 & 1.618 & 1.0000 & 1.5820 & $-2.2 \%$ \\
\hline
\end{tabular}


Table 8. Numerical results of Lai and Kulacki [22] and the film model for the effect of transpiration on free convective heat transfer to a horizontal plate ( $q_{\mathrm{w}}$ is constant)

\begin{tabular}{cccccc}
\hline \multicolumn{2}{c}{ Lai and Kulacki [22] } & $\begin{array}{c}\text { equation (20) } \\
\Theta_{\mathrm{t} \text {.hnc }}\end{array}$ & $\begin{array}{c}\text { equation (21) } \\
\phi_{\mathrm{t} \text {,hnc }}\end{array}$ & $\begin{array}{c}\text { equation (7) } \\
\Theta_{\mathrm{t}, \mathrm{film}}\end{array}$ & $\begin{array}{c}\text { equation (22) } \\
E_{\mathrm{hnc}}\end{array}$ \\
\hline-0.8 & 0.6835 & 0.8412 & -0.4923 & 0.7740 & $-8.0 \%$ \\
-0.4 & 0.7433 & 0.9148 & -0.2462 & 0.8819 & $-3.6 \%$ \\
0.0 & 0.8125 & 1.0000 & 0.0000 & 1.0000 & $0.0 \%$ \\
0.2 & 0.8510 & 1.0474 & 0.1231 & 1.0628 & $1.5 \%$ \\
0.4 & 0.8923 & 1.0982 & 0.2462 & 1.1281 & $2.7 \%$ \\
0.6 & 0.9366 & 1.1527 & 0.3692 & 1.1959 & $3.7 \%$ \\
0.8 & 0.9839 & 1.2110 & 0.4923 & 1.2663 & $4.6 \%$ \\
1.0 & 1.0344 & 1.2731 & 0.6154 & 1.3391 & $5.2 \%$ \\
\hline
\end{tabular}

Table 9. Numerical results of Lai and Kulacki [22] and the film model for the effect of transpiration on free convective heat transfer to a horizontal plate ( $v_{\mathrm{w}}$ is constant)

\begin{tabular}{cccccc}
\hline \multicolumn{2}{c}{ Lai and Kulacki [22] } & & equation (20) & & equation (21) \\
$\boldsymbol{\phi}_{\mathrm{t}, \mathrm{hnc}}$ & $-\Theta^{\prime}(0)$ & $\begin{array}{c}\text { equation (7) } \\
\Theta_{\mathrm{t}, \mathrm{film}}\end{array}$ & $\begin{array}{c}\text { equation (22) } \\
E_{\mathrm{hnc}}\end{array}$ \\
\hline-0.8 & 1.3853 & 0.8822 & -0.5095 & 0.7668 & $-13.1 \%$ \\
-0.4 & 1.4688 & 0.9354 & -0.2547 & 0.8781 & $-6.5 \%$ \\
0.0 & 1.5702 & 1.0000 & 0.0000 & 1.0000 & $0.0 \%$ \\
0.2 & 1.6288 & 1.0373 & 0.1274 & 1.0651 & $2.7 \%$ \\
0.4 & 1.6920 & 1.0776 & 0.2547 & 1.1328 & $5.1 \%$ \\
0.6 & 1.7632 & 1.1229 & 0.3821 & 1.2032 & $7.2 \%$ \\
0.8 & 1.8421 & 1.1732 & 0.5095 & 1.2763 & $8.8 \%$ \\
1.0 & 1.9298 & 1.2290 & 0.6369 & 1.3520 & $10.0 \%$ \\
\hline
\end{tabular}

The mean Nusselt number is defined as :

$$
N u_{\mathrm{L}}=\frac{1}{L} \int_{x=0}^{L} N u_{x} \mathrm{~d} x .
$$

According to our film model, equation (27) in the case of a surface mass reads :

$$
N u_{\mathrm{L}}=\frac{1}{L} \int_{x=0}^{L} N u_{x}^{*} \Theta_{\mathrm{t}, \mathrm{film}} \mathrm{d} x,
$$

see equation (8).

For flow along impermeable plates, i.e. $\Theta_{\text {t.film }}=1$, the integral can be solved in most practical situations, see equations (10)-(14). For convection with mass transfer, i.e. $\Theta_{t, \text { tilm }} \neq 1$, the integral can be solved too in closed form if $\phi_{t}$, and hence $\Theta_{\mathrm{t}, \mathrm{film}}$, are constant. This is the case if a similarity solution of the governing equations is imposed, implying a constant $f_{\mathrm{w}}[20-22]$, see equations (21) and (25).

Accordingly, in the case of constant $f_{\mathrm{w}}$ and $\phi_{\mathrm{t}}$ the mean heat transfer coefficient simply follows from multiplying the mean neutral heat transfer coefficient by the (constant) film model correction factor $\Theta_{t, \text { film }}$.

\section{CONCLUSIONS}

In this paper a film model has been derived for and applied to convective heat transfer between a flowing fluid in a porous medium and an adjacent permcable wall. This film model yields a relatively simple correction factor for heat transfer $\Theta_{\mathrm{t}, \mathrm{fim}}$. In order to apply this correction factor one needs to know the neutral (or zero mass transfer) heat transfer correlation. In the third section available data on neutral Nusselt numbers are amply summarized. Next, the film model predictions have been extensively compared with existing theoretical results concerning heat transfer in the presence of suction or injection. These foregoing studies were based on boundary layer models and whereby the obtained set of self-similar equations was solved numerically.

The results illustrate the substantial effect of a surface mass flux on heat transfer. For an injection rate of $\phi_{1} \cong-0.5$ for instance, convective heat transfer is already reduced by about $20 \%$. For a fluid withdrawal level of $\phi_{\mathrm{t}} \cong 0.5$, heat transfer is enhanced by about $25 \%$. The film model appeared to correlate within $13.1 \%$ with the boundary layer model data for $\left|\phi_{t}\right| \leqslant 0.4537$. These values apply to mixed convective flow along horizontal plates, and pure forced and pure free convection along both vertical and horizontal plates (data on mixed convection about vertical permeable plates are not yet available). These problems and stated $\phi_{1}$ range extend well beyond a large number of practical applications.

Furthermore, it was demonstrated that for the selfsimilar situations considered, the mean Nusselt number in the case of wall mass transfer simply follows from multiplying the neutral (or impermeable wall) mean Nusselt number by the (constant) film model correction factor $\Theta_{\text {t.fim }}$.

Though attention was restricted here to flat hori- 
zontal and vertical plates, it is expected that the film model is applicable, with a comparable accuracy, to the intermediate situation of inclined surlaces.

Acknowledgements - The author wishes to express his gratitude to Professor H. van Tongeren for his encouragement of this work and the Cornelis Lely Foundation for its financial support.

\section{REFERENCES}

1. H. P. G. Darcy, Les Fontaines Publiques de la Ville de Dijon. Victor Dalmont, Paris (1856) (in French).

2. J. Boussinesq, Thérie Analytique de la Chaleur, Vol. 2. Gauthier-Villars, Paris (1903) (in French).

3. A. Oberbeck, Ueber die Wärmeleitung der Flüssigkeiten bei Berücksichtigung der Strömungen infolge von Temperaturdifferenzen, Ann. Phys. Chem. 7, 271-292 (1879) (in German).

4. D. D. Joseph, Stability of convection in containers of arbitrary shape, J. Fluid Mech. 47, 257-282 (1971).

5. C. W. Horton and F. T. Roger, Convection currents in a porous medium, J. Appl. Phys. 16, 367-370 (1945).

6. E. R. Lapwood, Convection of a fluid in a porous medium, Proc. Cambridge Phil. Soc. 44, 508-521 (1948).

7. R. A. Wooding, Steady state free thermal convection of fluid in a saturated permeable medium, J. Fluid Mech. 2, 273-285 (1957).

8. R. A. Wooding. Convection in a saturated porous medium at large Rayleigh number or Peclet number, $J$. Fluid Mech. 15, 527-544 (1963).

9. A. McNabb, On convection in a porous medium, Proc. 2nd Australian Conf. on Hydraulics and Fluid Mech. pp. Cl61-C171. The University of Auckland Press, New Zealand (1965).

10. M. Prats, The effects of horizontal fluid flow on thermally induced convection currents in porous mediums, $J$. Geophys. Res. 71, 4835-4837 (1966).

11. J. W. Elder, Steady free convection in a porous medium heated from below, J. Fluid Mech. 27, 29 - 48 (1966).

12. M. A. Combarnous and P. Bia, Combined free and forced convection in porous media. Soc. Petrol. Engrs. J. II, 399.405 (1971).

13. P. Cheng and I. D. Chang, Buoyancy induced flows in a saturated porous medium adjacent to impermeable horizontal surfaces, Int. J. Heat Mass Transfer 19, 1267I272 (1976)

14. P. Cheng and W. J. Minkowycz, Free convection about a vertical flat plate embedded in a porous medium with application to heat transfer from a dike, J. Geophys. Res. 82, 2040-2044 (1977).

15. P. Cheng, Combined free and forced convection flow about inclined surfaces in porous media, Int. $J$. Heat Mass Transfer 20, $807 \% 814$ (1977).

16. P. Cheng, Similarity solutions for mixed convection from horizontal impermeable surfaces in saturated porous media, Int. J. Heat Mass Transfer 20, 893-898 (1977).

17. K. J. Renken and D. Poulikakos, Experiments on forced convection from a horizontal heated plate in a packed bed of glass spheres, I. Heat Transfer 1 11, 59-65 (1989).

18. K. J. Renken and D. Poulikakos, Mixed convection experiments about a horizontal isothermal surface embedded in a water-saturated packed bed of spheres, Int. J. Heat Mass Transfer 33, 13701373 (1990).

19. F. C. Lai and F. A. Kulacki, Experimental study of free and mixed convection in horizontal porous layers locally heated from below, Int. J. Heat Mass Transfer 34, 525541 (1991)

20. P. Cheng. The influence of lateral mass flux on free convection boundary layers in a saturated porous medium, Int. J. Heat Mass Transfer 20, 201-206 (1977).

21. W. J. Minkowycz, P. Cheng and F. Moalem, The effect of surface mass transfer on buoyancy-induced Darcian flow adjacent to a horizontal heated plate, Int. Comm. Heat Mass Transfer 12, 55-65 (1985).

22. F. C. Lai and F. A. Kulacki, The influence of surface mass flux on mixed convection over horizontal plates in saturated porous media. Int. J. Heat Mass Transfer 33, $576.579(1990)$

23. A. C. Bannwart, Etude theoretique et expérimentale de la condensation d'une vapeur en présence d'incondensables, Thèse de Doctorat, Institut National Polytechnique de Grenoble (1988) (in French).

24. A. C. Bannwart and A. Bontemps, Condensation of a vapour with incondensables : an improved gas phase film model accounting for the effect of mass transfer on film thicknesses. Int. J, Heat Mass Transfer 33, 1465-1474 (1990).

25. H. J. H. Brouwers, Film models for transport phenomend with foy formation, with application to plastic heat exchangers and condensers, Ph.D. Thesis, Eindhoven University of Technology (1990)

26. H. J. H. Rrouwers and A. K. Chesters, Film models for transport phenomena with fog formation: the classical film model, Int. J. Heat Mass Transfer 35, 1-11, 2067 (1992).

27. H. S. Mickley, R. C. Ross, A. L. Squyers and W. E Stewart, Heat, mass, and momentum transfor for flow over a flat plate with blowing or suction, NACA Techn. Note 3208 (1954).

28. H. J. H. Brouwers, A film model for free convection over a vertical porous plate with blowing or suction, Wärmeund Stoffühertragung 29, 17-26 (1994).

29. H. J. H. Brouwers, The effect of blowing or suction on laminar free convective heat transfer on flat horizontal plates. Wärme-und Stoffübertragung 28, 341-344 (1993). 